\title{
Adolescent Immunization Schedule: Need for a Relook
}

\author{
VIPIN M VAShishtha \\ From Mangla Hospital and Research Center, Shakti Chowk, Bijnor, Uttar Pradesh, India. \\ Correspondence to: Dr Vipin M. Vashishtha, Director and Consultant Pediatrician, Mangla Hospital and Research Center, Shakti \\ Chowk, Bijnor, Uttar Pradesh, 246 701, India.vipinipsita@gmail.com
}

\begin{abstract}
Adolescent immunization is one of the important yet a neglected field in India. There is no adolescent-specific schedule in the government's Universal Immunization Program. Though a separate adolescent immunization schedule exists for the private sector, there is almost no data on the coverage rates of the adolescent vaccines. With the changing epidemiology of certain vaccine preventable diseases, rapid development in the field of vaccinology and the advent of some new vaccines, there is a need to revisit the adolescent vaccination schedule. Common vaccine preventable diseases like dengue, mumps, hepatitis A and typhoid should be given higher priority whereas an alternate strategy should be adopted on the use of the vaccine against diphtheria, tetanus and pertussis.
\end{abstract}

Keywords: Adolescence, Immunity, Universal Immunization Program, Vaccines.

A

dolescent vaccination is almost non-existent in India's Universal Immunization Program (UIP) [1]. Till very recently, the only disease targeted for prevention among the adolescents and adults was tetanus. However, with the recent launch of Measles-Rubella vaccination campaign [2], and the proposed substitution of Tetanus-Toxoid (TT) with Tetanus-diphtheria (low adult dose) (Td) vaccine, three more diseases, i.e. measles, rubella and diphtheria have joined tetanus as the vaccine preventable diseases (VPDs) targeted for prevention and control amongst adolescents. Japanese encephalitis vaccine is also offered to adolescents and adults, but only in endemic districts of few states.

There is a lack of systematic epidemiological data defining the exact burden of various diseases in adolescent period. The overall focus of the government is to ensure good coverage rates for the UIP vaccines to infants, and even the booster doses meant for older children are not given adequate attention [3]. Barring TT, there is no data on the coverage of the vaccines given to adolescents in India. According to WHO-UNICEF estimates for 2017, India has attained more than $99 \%$ coverage with two doses of TT vaccine among 10-16 years adolescents and pregnant women whereas the similar figures for the years 2016 and 2015 were $76 \%$ and $77 \%$ [4]. Since overall vaccination coverage of the vaccines given to infants and young children is low [3], most Indian adolescents are expected to be either having partial or no vaccination at all.

\section{SignifiCANCE OF AdOLESCENT IMMUNIZATION}

Immunization of adolescents is vital. Vaccines are offered to adolescents to protect them against the diseases that have higher morbidity (hepatitis A, varicella), or higher incidence (mumps, meningococcal infection) during adolescent period. It is also aimed at boosting the waning immune responses of certain vaccines administered during infancy/early childhood (measles, pertussis, tetanus, diphtheria, etc). With the success of UIP initiative, the average age of few VPDs like diphtheria [5] and measles [6], has shifted upward, which that has also made adolescents more susceptible to these diseases. The epidemiology of certain VPDs such as hepatitis A has changed due to improving economy, better sanitation, and personal hygiene resulting in higher attack rates in adolescents and adults [7]. Another indication of adolescent immunization is to provide protection against diseases like cervical cancer appearing during adulthood. Vaccination of adolescents has also become an important component of few VPDs control or elimination projects like Measles Elimination, and Rubella and Congenital Rubella Syndrome (CRS) Control Program [2]. Furthermore, the tendency of the adolescents to indulge in risk-taking activities like substance abuse, intravenous administration of drugs and promiscuity also exposes them to certain diseases like hepatitis B and human papilloma virus (HPV) infection [1].

\section{Prioritizing an Adolescent Immunization Schedule}

There is no adolescent-specific immunization schedule devised by the Government of India. However, for the private sector, the Indian Academy of Pediatrics (IAP) is having a separate adolescent immunization schedule that comprises of three broad categories, namely 'mandatory' (HPV and Tdap), 'catch-up' (MMR, Varicella, Typhoid, Hepatitis B and A), and vaccines given under 'special 
circumstances' (Influenza, Japanese Encephalitis (JE), PPSV and Rabies) [8]. The epidemiology of VPDs has changed in the last decade, owing to 'vaccine pressure' (JE), to changing lifestyle (Hepatitis A), or following good performance of UIP (diphtheria, measles, and pertussis). Few new diseases have also emerged as a major burden during the adolescent period (dengue). Some new and more efficacious vaccines against infectious diseases have become available (typhoid conjugate and dengue vaccines), and new insights on some other vaccines have come to the horizon (HPV, Tdap).

The IAP-ACVIP has recently revised its recommendations for 2018-19 immunization schedule for children aged 0 through 18 Years, but there is no separate section of adolescent immunization [9]. There is a need to revise categories and the prioritization of the adolescent vaccines in the immunization schedule. While HPV, Dengue, Mumps/MMR, Td/Tdap, Hepatitis A, Typhoid, Varicella and Hepatitis B vaccines should be recommended for all adolescents, Meningococcal, Pneumococcal polysaccharide vaccine (PPSV), JE, Influenza, Rabies, Cholera and Yellow fever vaccines should be given under special circumstances.

Following should be the appropriate order of the adolescent vaccines based on the severity of the disease, their significance to the adolescents and adults, and the burden and the risk of acquiring the infection during adolescent period (Box 1).

Human Papillomavirus (HPV) vaccine: This vaccine is an adolescent-specific vaccine, and should remain at the top of the priority list considering the significant burden of cervical cancer in India, accounting nearly $25 \%$ of global cervical cancer deaths. In 2012, there were 123,000 new cases of cervical cancer and 67,000 deaths in India [10]. The age-standardized incidence ratio for cervical cancer in India is 22.9 per 100,000 women per year, which is higher than the incidence in South East Asia (16.3 per 100,000) and in the world (14 per 100,000) [10]. Few states of India have already started pilot projects on HPV vaccination since 2016. With the availability of highly efficacious vaccines and a shortened 'two-dose schedule', which should be more affordable and compliant, the use of HPV vaccines should be encouraged.

Dengue vaccine: Dengue has recently become a VPD with the advent of a live recombinant tetravalent dengue vaccine. Dengue, the fastest spreading viral infection in the world, is also the number one concern not only for India but to entire South-East Asia (SEA) region. Dengue poses a substantial economic and disease burden which is higher than that of 17 other conditions, including JE and hepatitis B [11]. Though dengue affects all age
Box 1 Suggested Prioritization of Vaccines Administered to Adolescents IN INDIA

- HPV vaccine

- Dengue vaccine

- Tetanus-diphtheria(Td) vaccine

- Mumps/MMR vaccine

- Hepatitis-A vaccine

- Typhoid vaccine

- Varicella vaccine

- Hepatitis-B vaccine

groups, it is primarily a disease of adolescents and adults. According to recent reports, the majority of dengue cases occur in the age group of 14-45 years with highest burden seen in the 15-24 years sub-group [12]. Unfortunately, the only globally licensed vaccine (Dengvaxia) has got few limitations like poor immune response against Type 2 strain of dengue virus, moderate efficacy, multi-dose schedule, effective only in the age group of 9-45 years, and most importantly, a real risk of developing the severe disease in seronegative individuals. [13]. Nevertheless, the WHO has approved its use in the highly endemic countries where around $90 \%$ of the population may be infected with the dengue virus by adolescence. These seropositive individuals would clearly benefit from receiving the vaccine. Although the WHO still believes that the public health and economic benefits of the Dengue vaccine far outweigh the potential risks [13], it would be a risky proposition to employ this vaccine in any large-scale immunization program like UIP in India. Fortunately, the pipeline for new, superior dengue vaccines seems quite robust, and at least two candidate vaccines are in the phase III trial stage. The licensure of one of them (Takeda's TDV dengue vaccine) for clinical use looks imminent [14]. Indian companies are also involved in developing new dengue vaccines [15]. With the availability of more 'refined' dengue vaccines, its integration in the list of essential adolescent vaccines would make sense. With some newer vaccines having a two-dose schedule, a combination of HPV and dengue vaccine can be implemented in different settings like in schools, health facilities and outreach sessions.

Tetanus-diphtheria-acellular pertussis (Tdap) and Tetanus-diphtheria (Td) vaccine: Tdap along with HPV vaccine is so far considered as a 'mandatory' adolescent vaccine. However, with the recent incidents of recurrent outbreaks of pertussis in vaccinated adolescents has raised doubts about the utility of Tdap against pertussis. World over, the experts are now opining to adopt an 
alternate strategy to use Tdap in anticipation of a local pertussis outbreak rather than on a routine basis in adolescents as it provides only a short-term protection against pertussis [16]. Recent studies have shown that Tdap vaccine effectiveness decreases with the passage of time, and protection wanes rapidly after $1-2$ years [1619]. In one large study, the efficacy of Tdap against pertussis component had waned to a meagre $8.9 \%$ after 4 years [16].

There is no data on the burden of pertussis in adolescents in India. Since the data on the efficacy/ effectiveness of the Tdap from our country and other South Asian countries are sorely lacking, it would be more prudent to reserve its use to protect an adolescent during an ongoing outbreak or when an outbreak is anticipated rather than using it routinely for all adolescents. Another key indication of Tdap would be to use it during pregnancy to protect very young infants from pertussis. Hence, Tdap should be substituted with bivalent tetanusdiphtheria (Td) vaccine which would not only be a more judicious but also a cost-effective strategy.

Mumps vaccine: Mumps is yet another vaccine that needs to be pushed up in the schedule. The highest incidence of mumps in India is seen in children above 5 years of age, mostly in the adolescent age group [20]. While there is a considerable waning of immunity following mumps vaccination and 'time since vaccination' seems to be a factor that determines the risk of outbreaks in adolescents, a dose of mumps/MMR at 46 yrs may not be effective in preventing outbreaks amongst older adolescents aging 15-18 years. Since the first dose of MMR is now offered before 12 months of age in private sector when a robust immune response may not be elicited [9], the need for additional doses becomes all the more important. Whether the third dose of the vaccine be delayed to adolescent period is a debatable issue. At least, a single-dose of mumps/MMR is a must for every adolescent irrespective of their past vaccination status.

Hepatitis A vaccine: Incidence of hepatitis A virus (HAV) infection in India is shifting from high endemicity to intermediate endemicity [7]. This has resulted in pushing up the average age of infection, and consequently, has increased the proportion of infections that result in severe disease during adolescents and adults. Studies in India have shown HAV seroprevalence to be between $38 \%$ to $92 \%$ in different age groups; a higher attack rate was seen in adolescents and adults than young children $(4.6 \%$ vs. $3.1 \%)$ in some Indian studies [21]. With outbreaks of HAV infection, higher proportions of symptomatic cases and even hepatic failures are reported among adolescents from different parts of the country [7].
Due to their tendency of more frequent visits to restaurants and eating outside the home, adolescents are more vulnerable to acquire HAV infection. Hepatitis A vaccination should be given a high priority for catch-up immunization of adolescents.

Typhoid vaccine: India and few other South-east Asian countries have got a very high incidence of typhoid fever. Children aged 5-15 years and adolescents are at greatest risk of developing the disease [22]. According to Global Burden of Disease Study, in 2016, India had 6.6 million typhoid cases (499 cases per 100,000 population) and 66,439 typhoid deaths, $56 \%$ of which were among children under 15 years of age [23]. A study of typhoid fever in five Asian countries found the highest incidence of typhoid in the age group of 5-15 years ( 493.5 cases per 100000 /year) followed by 2-4 years age group (340.1 cases per 100,000/year) at the Indian site [24]. Like Hepatitis A, adolescents are more vulnerable to catch typhoid infection; therefore, they should be targeted for vaccination.

Varicella vaccine: The epidemiology of Varicellazoster virus (VZV) infection is different in tropical countries in which VZV infection is common in adolescents and adults than in temperate countries where adolescents and adults are almost immune with universal seroconversion occurring by late childhood [25]. The varicella disease is also a far more severe ailment with greater morbidity and mortality in adolescents and adults than in early childhood. Furthermore, VZV infection during pregnancy may have serious health hazards for the fetus and newborn infant.

Hepatitis $B$ vaccine: India is now considered among the category of intermediate endemicity for hepatitis B and its prevalence is found to be $2-7 \%$, with an average of $4 \%$ [26]. Although the predominant mode of transmission of hepatitis B in India is horizontal, perinatal transmission through mother to child is also not insignificant. Adolescents are more vulnerable to get the hepatitis B infection through indulgence in sexual activities which also facilitates its transmission. Thus, vaccination of adolescents against hepatitis $\mathrm{B}$ becomes imperative.

\section{Conclusion}

With the renewed thrust provided by the GAVI for introduction of new vaccines like HPV and target elimination of certain VPDs like measles and rubella, the adolescent vaccination cannot be neglected for far too long. It is imperative to revisit existing immunization schedule for adolescents considering the rapid changes occurring in the field of immunization and epidemiology of few VPDs. This may provide adequate 'signals' to the 
UIP managers to give more serious thoughts to adolescent immunization.

Funding: None; Competing interest: None stated.

\section{REFERENCES}

1. Verma R, Khanna P, Chawla S. Adolescent vaccines: Need special focus in India. Hum Vaccin Immunother. 2015; $11: 2880-2$.

2. World Health Organization. India's Measles Rubella Vaccination Campaign a Big Step Towards Reducing Childhood Mortality, Addressing Birth Defects; 2016. Available from: http://www.searo.who.int/mediacentre/ features/2017/india-measles-rubella-vaccinationcampaign/en/. Accessed August 30, 2018.

3. National Family Health Survey, India. NFHS-4 Publications. Available from: http://rchiips.org/nfhs/ NFHS-4Reports/India.pdf. Accessed March 4, 2018.

4. World Health Organization. Reported estimates of TT2+ coverage. Available from: http://apps.who.int/ immunization_monitoring/globalsummary/timeseries/ tscoveragett2plus.html. Accessed August 30, 2018.

5. Murhekar M. Epidemiology of diphtheria in India, 19962016: Implications for prevention and control. Am J Trop Med Hyg. 2017;97:313-8.

6. Bajaj S, Bobdey P, Singh N. Measles outbreak in adults: A changing epidemiological pattern. Medical Journal of DY Patil University. 2017;10:447-52.

7. Arankalle V, Mitra M, Bhave S, Ghosh A, Balasubramanian $\mathrm{S}$, Chatterjee $\mathrm{S}$, et al. Changing epidemiology of hepatitis A virus in Indian children. Vaccine: Development and Therapy. 2014:4:7-13.

8. Choudhury P. Immunization of Adolescents. In: Vashishtha VM, Choudhury P, Bansal CP, et al. IAP Guidebook on Immunization 2013-2014. Gwalior: National Publication House, Indian Academy of Pediatrics; 2014; 357-63.

9. Balasubramanian S, Shah A, Pemde HK, Chatterjee P, Shivananda S, Guduru VK, et al. Indian Academy of Pediatrics (IAP) Advisory Committee on Vaccines and Immunization Practices (ACVIP) Recommended Immunization Schedule (2018-19) and Update on Immunization for Children Aged 0 Through 18 Years. Indian Pediatr. 2018;55:1066-74.

10. Kaur P, Mehrotra R, Rengaswamy S, Kaur T, Hariprasad $\mathrm{R}$, Mehendale SM, et al. Human papillomavirus vaccine for cancer cervix prevention: Rationale and recommendations for implementation in India. Indian $\mathrm{J}$ Med Res. 2017;146:153-7.

11. Shepard DS, Undurraga EA, Halasa YA. Economic and disease burden of dengue in Southeast Asia. PLoS Negl Trop Dis. 2013;7:e2055.

12. Mutheneni SR, Morse AP, Caminade C, Upadhyayula SM. Dengue burden in India: Recent trends and importance of climatic parameters. Emerg Microbes Infect. 2017; $6: \mathrm{e} 70$.
13. World Health Organization. Meeting of the Strategic Advisory Group of Experts on Immunization, April 2018 Conclusions and Recommendations. Wkly Epidemiol Rec. 2018,93;329-44.

14. Wichmann O, Vannice K, Asturias EJ, de Albuquerque Luna EJ, Longini I, Lopez AL, et al. Live-attenuated tetravalent dengue vaccines: The needs and challenges of post-licensure evaluation of vaccine safety and effectiveness. Vaccine. 2017;35:5535-42.

15. Dengue Fever Vaccine Pipeline Update: The Latest Progress on Protection Against the Virus. Available from: https://www.breakdengue.org/dengue-fever-vaccinepipeline/. Accessed March 10, 2018.

16. Klein NP, Bartlett J, Fireman B, Baxter R. Waning Tdap effectiveness in adolescents. Pediatrics. 2016;137: e20153326.

17. Koepke R, Eickhoff JC, Ayele RA, Petit AB, Schauer $\mathrm{SL}$, Hopfensperger DJ, et al. Estimating the effectiveness of tetanus-diphtheria-acellular pertussis vaccine (Tdap) for preventing pertussis: evidence of rapidly waning immunity and difference in effectiveness by Tdap brand. J Infect Dis. 2014;210:942-53.

18. Acosta AM, DeBolt C, Tasslimi A, Lewis M, Stewart LK, Misegades LK, et al. Tdap vaccine effectiveness in adolescents during the 2012 Washington State pertussis epidemic. Pediatrics. 2015;135:981-9.

19. Briere EC, Pondo T, Schmidt M, Skoff T, Shang N, Naleway A, et al. Assessment of Tdap Vaccination effectiveness in adolescents in integrated health-care systems. J Adolesc Health. 2018; 62:661-6.

20. Vashishtha VM, Yadav S, Dabas A, Bansal CP, Agarwal $\mathrm{RC}$, Yewale VN, et al. IAP position paper on burden of mumps in India and vaccination strategies. Indian Pediatr. 2015; 52:505-14.

21. Rathi A, Sharma S. Vaccine preventable diseases in indian adults-burden and prevention. Infect Dis Diag Treat. 2017:J102.

22. Antillón M, Warren JL, Crawford FW, Weinberger DM, Kürüm E, Pak GD, et al. The burden of typhoid fever in low-and middle-income countries: A meta-regression approach. PLoS Negl Trop Dis. 2017;11:e0005376.

23. GBD 2016 Causes of Death Collaborators. Global, regional, and national age-sex specific mortality for 264 causes of death, 1980-2016: A systematic analysis for the Global Burden of Disease Study 2016. Lancet. 2017;390:1151-210.

24. Ochiai RL, Acosta CJ, Danovaro-Holliday MC, Baiqing D, Bhattacharya SK, Agtini MD, et al. A study of typhoid fever in five Asian countries: disease burden and implications for controls. Bull World Health Organ. 2008;86:260-8.

25. Lee BW. Review of varicella zoster seroepidemiology in India and Southeast Asia. Trop Med Int Health. 1998;3:886-90.

26. Ray G. Current scenario of hepatitis $B$ and its treatment in India. J Clin Transl Hepatol. 2017;5:277-96. 